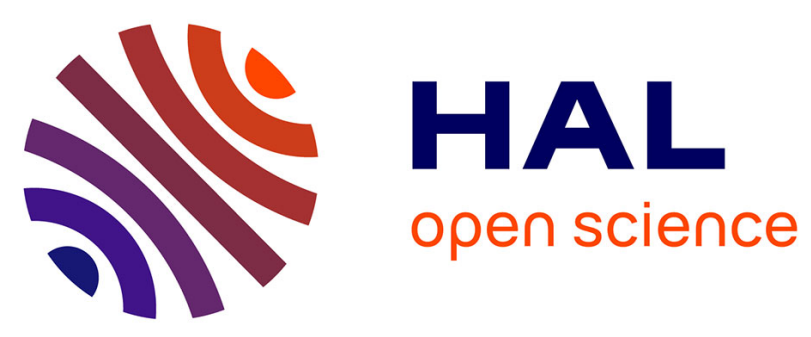

\title{
Essai sur le don de gamètes
}

Claire Squires

\section{- To cite this version:}

Claire Squires. Essai sur le don de gamètes. Topique - Revue freudienne, 2011, 10.3917/top.116.0073 . hal-01451281

\section{HAL Id: hal-01451281 \\ https://hal.science/hal-01451281}

Submitted on 31 Jan 2017

HAL is a multi-disciplinary open access archive for the deposit and dissemination of scientific research documents, whether they are published or not. The documents may come from teaching and research institutions in France or abroad, or from public or private research centers.
L'archive ouverte pluridisciplinaire HAL, est destinée au dépôt et à la diffusion de documents scientifiques de niveau recherche, publiés ou non, émanant des établissements d'enseignement et de recherche français ou étrangers, des laboratoires publics ou privés. 


\title{
ESSAI SUR LE DON DE GAMèTES
}

\author{
Claire Squires
}

\section{L?Esprit du temps I Topique}

\section{$2011 / 3-n^{\circ} 116$ \\ pages 7388}

\section{ISSN 0040-9375}

Article disponible en ligne l'adresse:

http://www.cairn.info/revue-topique-2011-3-page-73.htm

Pour citer cet article :

Squires Claire, Essai sur le don de gamètes,

Topique, $2011 / 3 \mathrm{n}^{\circ} 116$, p. $73-88$. DOI : 10.3917/top.116.0073

Distribution ectronique Cairn.info pour L?Esprit du temps.

L?Esprit du temps. Tous droits rerv pour tous pays.

La reproduction ou reprentation de cet article, notamment par photocopie, n'est autoris que dans les limites des conditions gales d'utilisation du site ou, le cas hnt, des conditions gales de la licence souscrite par votre ablissement. Toute autre reproduction ou reprentation, en tout ou partie, sous quelque forme et de quelque manie que ce soit, est interdite sauf accord prlable et rit de l'iteur, en dehors des cas prus par la lislation en vigueur en France. II est prisque son stockage dans une base de donns est alement interdit. 


\title{
Essai sur le don de Gamètes
}

\author{
Claire Squires
}

En France, les dons de spermatozoïdes puis d'ovocytes, plus récemment d'embryons, sont pratiqués depuis trente-cinq ans et ont abouti à la naissance de 40000 enfants issus de ces techniques. Si la conception par don est généralement acceptée du public qui souhaite vivement remédier aux infertilités, aux dires des couples rencontrés à la banque de gamètes (CECOS), elle reste encore mal connue, voire gênante, comme si elle n'intéressait que lorsqu'on est confronté au problème personnellement ou en raison de l'entourage.

Les débats autour de la levée de l'anonymat des dons de gamètes restent toujours émotionnels voire passionnels et les pratiques dans ce domaine n'ont nullement fait l'objet d'études sérieuses en France. La révision des lois de bioéthique de 2004 cette année ouvre à nouveau la discussion des principes initiés en France pour les dons de gamètes: anonymat des donneurs et gratuité des dons. Or certains pays ont décidé de lever l'anonymat du donneur partiellement ou complètement. L'Allemagne et la Suisse considèrent le droit à connaître ses origines génétiques, garanti par la Constitution ${ }^{1}$. La France, l'Espagne, la Norvège imposent dans la loi l'anonymat du tiers donneur de gamètes et la

1 - Depuis 1989, la Cour constitutionnelle allemande estime que ce droit constitue la conséquence logique des droits au libre épanouissement, ainsi que du principe d'égalité entre enfants légitimes et enfants naturels, eux-mêmes établis par la Loi fondamentale. En Suisse, depuis 1992, la Constitution fédérale comporte plusieurs dispositions relatives à la bioéthique, l'une d'elle énonçant: «L'accès d'une personne aux informations relatives à son ascendance est garanti.» 
gratuité totale ou partielle. ${ }^{2}$ Le débat bioéthique français se politise à gauche comme à droite en période électorale.

Les medias traitant pêle-mêle des dons pour des raisons médicales de stérilité et des dons chez les homosexuels, chez les célibataires pour des raisons sociétales, des grossesses pour autrui donnent l'impression d'une tension hystérique sans abord des changements sociétaux profonds créés par cette filiation élective avec dons de gamètes (non naturelle). Or, le silence et la prudence des cliniciens face aux bouleversements suscités par cette offre «procréatique » interrogent encore plus les enjeux psychiques immédiats et à plus long terme de ces procréations et chez les enfants ainsi conçus.

\section{LA «VÉRITÉ» BIOLOGIQUE ET LE DROIT DANS LES FILIATIONS ÉLECTIVES}

La place grandissante de la biologie en médecine de la reproduction a changé la façon de concevoir et de mettre au monde les enfants. Depuis l'avènement de la contraception ceux-ci, devenus rares, sont fortement investis. Aujourd'hui les couples ont un «projet d'enfant» alors que les incertitudes entourant autrefois la procréation donnaient aux rencontres une part de hasard et d'indécidable. De plus, depuis le recul de la mortalité infantile, les couples ont moins d'enfants qui deviennent aussi plus précieux. C'est pourquoi, l'infertilité relative ou complète est vécue comme une calamité, une injustice, une infirmité. «Vouloir un enfant » devient un impératif ressenti par le couple s'engageant dans des traitements longs, pénibles et coûteux.

Les techniques d'aide à la procréation introduisent une dissociation entre la sexualité et la procréation. Mais accentuant encore la part grandissante de la biologie dans la médecine de la reproduction, la quête de la vérité biologique de la part d'enfants issus de dons, peu nombreux mais militants, se développe. Ces enfants devenus majeurs souhaitent disposer d'informations sur le donneur ou pouvoir le rencontrer. Ce courant fait aussi partie d'une revendication à la connaissance de leurs origines issue de la Convention des droits de l'enfant à Genève. Le mouvement en faveur de la levée de l'anonymat du donneur s'inscrit dans le courant initié par des recherches en paternité ou en maternité visant à la « traçabilité » des origines, aboutissant à des remises en question de la filiation et

2 - En France, la levée partielle ou totale de l'anonymat des donneurs à la majorité de l'enfant né par don de gamètes a été refusée à l'assemblée et sera rediscutée en 2013 à la suite du passage au Sénat cette année. En Grande-Bretagne, où le don est rémunéré et le contexte socioculturel différent, les enfants conçus après avril 2005 pourront à l'âge de 18 ans et 9 mois demander l'identité de leur père biologique. Malgré la levée de l'anonymat du don en 2005, on ne constate pas dans ce pays de diminution du nombre de donneurs. Day M. Number of sperm donors rises despite removal of anonymity in UK. BMJ. 2007 May 12; 334 (7601): 971. 
une fragilisation des liens de l'enfant. Il est aujourd'hui possible d'obtenir auprès de laboratoires spécialisés privés une réponse quasi immédiate, quoique peu fiable, à l'aide de quelques cellules lors d'une recherche en paternité ou maternité de son père ou de sa mère. Savoir si son père ou sa mère sont bien ses parents biologiques sera de plus en plus facile et en la matière, il n'y aura plus grand secret. La «vérité » biologique reste le critère ultime en cas de contestation des filiations. Ne risque-t-on pas de s'enfermer dans cette recherche génétique des origines?

Sur le plan juridique, la filiation est d'abord établie par volonté des parents, soit dans le mariage, soit dans la reconnaissance. La parenté et la filiation juridiques ne se définissent pas comme la vérité biologique mais en référence à cette dernière. Les parents sont ceux qui ont engendré l'enfant ou sont considérés comme tels. La particularité du système juridique actuel concernant les procréations avec dons de gamètes réside dans le décalque quasi parfait de ces filiations électives sur une filiation biologique. Pour éviter indiscrétions et discriminations, tout est fait pour que ces filiations purement juridiques aient l'allure de filiations biologiques. Or, les effets de la filiation sont importants et structurants tant pour les individus que pour la société et pour certaines filiations électives, des enfants étant rattachés juridiquement à des couples qui ne sont pas leurs parents biologiques.

Il y a donc dans la filiation juridique une dimension biologique mais elle est loin d'être la seule et d'avoir une valeur absolue. Mais, ce qui choque et crée un malaise ou une incompréhension, c'est le refus de tenir compte du lien biologique dans certains cas comme l'assistance médicale à la procréation, l'accouchement «sous $\mathrm{X}$ » et l'adoption, alors qu'on la tient pour référence dans d'autres, comme la recherche judiciaire de paternité. Ainsi la prégnance du biologique retentit sur les nombreuses formes d'adoptions qu' on connaît depuis le droit romain en Occident.

Pourquoi la médecine de la reproduction accorderait-elle, presque malgré elle tant de place à la vérité biologique dans les cas de recherche de filiation et favoriserait l'aspect relationnel des relations familiales en cas de dons de gamètes ? Comment à un moment où chacun peut disposer de connaissances sur son génome empêcherait-elle des enfants d'y avoir accès et de satisfaire une question qu'on accorde à des parents voulant contester la filiation de leur enfant ?

\section{«L'ENFANT DU DÉSIR »}

Mais par-delà les aspects biologiques et juridiques, la naissance est un événement certes situé dans le corps mais aussi dans une parole et une culture qui façonnent l'art de «fabriquer les enfants » et celui de traiter les aléas de la procréation. Ainsi Pierre Levy-Soussan s'interroge, dans la Revue française de 
psychanalyse, «sur le processus qui permet de transcender le statut du biologique pour permettre à un individu de devenir parent de l'enfant et d'être reconnu à cette place par l'enfant, sa famille et la société. La fonction du couple dans cette perspective de dépassement du biologique paraît importante, non seulement dans son rôle de parent mais aussi comme lieu historique et historicisant à travers sa rencontre, sa sexualité, son asymétrie» (2002).

De plus, la naissance de l'enfant est entourée de tous les soins, une grande attention est portée aux premières années, aux émotions, aux liens d'intersubjectivité, à l'attachement avec la construction d'une famille. Les familles se concentrent sur les débuts de la vie alors que les liens conjugaux et les unions sont aussi plus fragiles. De telle sorte qu'en cas de difficulté à enfanter et de recours aux traitements médicaux, l'anticipation autour de l'enfant à naître est intense et angoissante. L'obligation de réussir est contraignante à la fois pour les parents et les médecins qui les accompagnent et les psychanalystes qui recueillent dans les entretiens avec les couples les tensions de cette quête d'espoirs et de déceptions.

Lorsque la technique médicale est au centre du dispositif et que l'enfant n'est pas issu du corps à corps de la rencontre amoureuse comment se représenter la part de «nature » dans la procréation? Et quelle est la part de «culture » lorsque la filiation est surtout sociale, affective et relationnelle ?

Jean-Claude Ameisen, immunologiste, discutait aux journées nationales d'éthique organisées par le Comité Consultatif National d'Éthique (CCNÉ) en janvier 2011 les bouleversements des valeurs entourant l'engendrement et l'enfantement:

«Comment voyons-nous ce que nous appelons « la nature» dans ces recompositions anthropologiques et culturelles ? Dans quelle mesure «le biologique» nous contraint avec ses avancées? Jusqu'où ce que nous croyons être la fonction de « la nature » doit nous servir de guide? Jusqu' où nos constructions culturelles doivent être fidèles à notre façon de voir « la nature?»

Nature (au sens de Rousseau par opposition à la société injuste et corrompue) et culture apparaissent indissociables et sculptées l'une par l'autre mais comment se représenter psychiquement la filiation et la procréation au regard des changements d'ordre biologique, juridique et relationnel?

L'enfant lui se reconnaît dans le récit de ses parents et de sa famille lequel est en partie validé par la société. Il reçoit émotionnellement les mots, les soins, l'attention, les traductions, les transmissions, les conceptions, l'héritage de ses parents, leurs identifications à leurs propres parents, ascendants, à des figures tutélaires, à des proches et aussi de tout l'infraverbal et les silences. Les parents véhiculent un sens, des significations de l'histoire où chacun peut s'inscrire. Ils sont aussi troublés par les débats qui traversent la société quant à la filiation et aux fantasmes qui s'y déchaînent («droit à l'enfant», «droit à la connaissance des origines ») et ont parfois du mal à y retrouver leurs questions personnelles. 
Le sens transmis des parents comporte une affectivité, une sexualité, une sensualité mais il y a aussi le sens caché, l'oubli, le refoulé, le travail du négatif. À partir de sa propre pulsionnalité et des interdits qui la balisent, de ses observations de la différence des sexes et des générations, de l'origine de filiation, de la construction de ses identifications et contre-identifications, l'enfant constitue son identité et son lien de filiation à partir des constructions œdipiennes et de son économie narcissique et invente son propre roman familial marqué par ses idéaux narcissiques.

Ainsi, concernant les dons de gamètes, si la biologie semble donner une possibilité technique pour permettre à l'enfant d'exister lorsque le couple ne peut enfanter, c'est le sens de cette procréation qui importe, la signification qu'ils lui donnent, en partie à leur insu aussi, afin que l'affiliation puisse exister. Ils pourront s'approprier la relation qu'ils construiront avec cette vérité qui est la leur et la co-construiront ensuite en étant reconnus par l'enfant (Lebovici, 2004). Le récit de cette histoire pourra être transmis en mots et en actes plus tard à l'enfant ou pas puisqu'ils en ont le choix intime jusqu'à présent.

Avec l'intrusion de la médecine au cœur de la scène primitive, dissociée de la reproduction sexuelle, il n'est pas étonnant que la transmission de la vie et surtout la façon de se la représenter en soit toute bouleversée. Il n'est pas plus facile d'être l'enfant d'un si fort désir semé d'embûches pour exister que d'être parents ne pouvant se passer de la médecine pour l'enfanter.

Au-delà de la question des procréations par dons, Marcel Gauchet (2010) débattant des incidences des mutations dans la famille se demande, notamment, ce qu'il en résulte pour l'enfant d'être désiré et de naître «l'enfant du désir»? Quel est le statut de ces enfants aujourd'hui si désirés? Ne sont-ils pas lestés de toute cette désirance parentale? Auparavant, avoir des enfants au sein de la « famille institutionnelle » consistait surtout à les élever et qu'ils deviennent autonomes : «L'enfant du désir est l'enfant de la famille privée, intimisée, désinstitutionnalisée, informelle, qui n'a d'autre raison d'être que l'épanouissement affectif de ses membres. On fait un enfant non pour la société, pour la perpétuation de l'existence collective, mais pour soi et pour lui-même.» L'auteur se demande si, compte tenu de l'environnement, l'enfant ne peut que se poser la question de son adéquation avec le désir de ses parents (par exemple: «Ai-je vraiment été désiré ?» ou bien «Suis-je l'enfant qui correspondait au désir de mes parents?» Ne trouvant pas de réponse à cette question existentielle en dehors d'une solution délirante, Marcel Gauchet invite très finement à se demander comment se constitue alors le roman familial de ces enfants du désir et s'ils ne sont pas rongés par le doute existentiel car la question du choix d'exister est insoluble, puisque donnée par la nature. Comment dès lors pourraient-ils accéder à l'individuation psychique? 


\section{LA SCÈNE PRIMITIVE DÉVOILÉE}

Des entretiens psychologiques proposés le plus souvent lors des demandes de procréation médicalement assistée par dons de gamètes (ils font partie du code de santé publique), devraient théoriquement permettre aux futurs parents d'élaborer les enjeux d'une telle conception, afin de se sentir mieux fondés comme parents, inscrits dans une filiation à la fois sociale et juridique, susceptibles de pouvoir éventuellement transmettre quelque chose de leur démarche à leur enfant. Mais ces entretiens sont ponctuels et interviennent juste au moment de la demande de gamètes, de telle sorte que leur effet psychique est une petite comète dans une nébuleuse de problèmes qu'ils ont du mal à subjectiver. La souffrance psychique liée à l'infertilité est peu considérée et traitée au moment de l'annonce de l'azoospermie ou d'une ménopause précoce, de telle sorte que de nombreux couples se retrouvent, seuls, à prendre connaissance des chiffres de spermatozoïdes ou des dosages hormonaux inscrits un papier du laboratoire. En effet, lors de ces consultations psychologiques au CECOS, confondues avec l'agir médical, vaincre l'infertilité est au premier plan de leurs préoccupations, l'absence de conception menaçant le couple. Il n'est pas évident de se démarquer comme psychanalyste du médical lorsque le traitement médical vient colmater leurs blessures et apporter une solution palliative. Lorsqu'une grossesse par don de gamètes s'annonce, il n'y a plus de centre de référence, pas de possibilité de suivi psychologique des couples et de leurs enfants pour élaborer ces questions. Pour les personnes souhaitant effectuer un don, un entretien permet de connaître les motivations conscientes (donner pour aider des amis, don altruiste, don parce qu'on a soi-même été aidé lors d'une procréation médicalement assistée par exemple, don d'ovocyte d'une femme qui a eu des enfants antérieurement par un don de spermatozoïdes) ; comment aller plus loin ?

La conception dans un contexte de dons de gamètes suscite des représentations spécifiques autour de la filiation et du fait de devenir parents alors qu'un des parents est privé de lien génétique avec l'enfant. Or les différents plans de la parentalité, à la fois affective, psychique, juridique, sociale et anthropologique ne sont guère abordés, alors même que les couples qui font la demande de dons de gamètes sont pris dans l'action plus que dans la réflexion.

Le Code de santé publique et la loi de bioéthique donnent un cadre pour les indications médicales de la stérilité puisqu'ils stipulent que l'assistance médicale à la procréation est destinée à répondre à la demande parentale d'un couple et «a pour objet de remédier à l'infertilité dont le caractère pathologique a été médicalement diagnostiqué. Elle peut avoir pour objet d'éviter la transmission à l'enfant une maladie d'une particulière gravité » (article L 152-2 du Code de la Santé publique, introduit en 1994).

L'AMP est aussi encadrée par des normes sociales puisqu'elle s'adresse à des couples mariés ou vivant ensemble depuis au moins deux ans. «L'AMP est 
destinée à répondre à la demande parentale d'un couple», elle sera précédée d'entretiens préalables par une équipe pluridisciplinaire dont les membres de l'équipe médicale devront «vérifier la motivation de l'homme et de la femme formant le couple et les informer des dispositions existantes en matière d'adoption» (article 152-10). Ainsi la loi permet qu'un entretien psychologique soit proposé au couple pour préciser sa demande d'enfant. Le Code de la santé rend obligatoire l'entretien psychologique pour le receveur dans le cas d'un don de gamètes, entretien avec un psychiatre ou un psychologue: «Le doute sérieux sur la santé psychique des demandeurs ou la validité de leurs motivations pourra conduire, après entretien avec le psychiatre ou le psychologue, à un délai de réflexion supplémentaire ou à ne pas accepter la demande.»

Comment vérifier la motivation des couples, authentifier en quelque sorte leur désir d'enfant, comme le précise la loi, s'assurer que la grossesse ne risquera pas de faire décompenser l'un des conjoints ? Qui doit prendre la responsabilité de l'acte médical, le gynécologue, le psychologue, le psychiatre ou le bureau responsable de l'éthique médicale ? S'agit-il d'un engagement personnel ou professionnel ou de la décision d'une équipe? Les psychanalystes savent bien qu'au discours explicite: «Je veux un enfant», se mêlent des motivations plus souterraines, plus ambivalentes, plus nuancées, des obstacles inconscients. Un parcours aussi contraignant que l'assistance médicale à la procréation laisse peu de place au flottement ou à l'indécision d'un des partenaires. D'un côté, pour mieux cerner les risques d'achoppement, il faudrait ne pas trop se hâter de proposer des solutions purement médicalisées. Et de l'autre, toute intervention supplémentaire d'un tiers peut être vécue comme intrusive.

Sylvie Epelboin, gynécologue à l'hôpital Saint-Vincent-de-Paul, souligne la difficulté à aborder les questions du désir d'enfant et de la sexualité du couple: «Dans l'investigation menée sur le contexte du projet d'enfant, nous sommes partagés entre deux volontés : ne pas passer à côté d'un problème majeur au sein duquel le désir d'enfant serait de colmater une brèche, mais aussi celle de ne pas obliger un couple à subir un «examen de passage». Sous prétexte que l'enfant désiré n'est pas venu dans un parcours naturel ou un délai souhaité, le couple se devrait de prouver la véracité de son désir et dévoiler les secrets de son intimité. L'obligation, pour ces couples, de présenter un projet construit, limite leur droit légitime à l'ambivalence.» (Epelboin, 1999, p. 89). Il n'est pas non plus souhaitable que la question du désir d'enfant soit expulsée de la consultation, comme le souligne Dominique Memmi (2001). 


\section{DON ANONYME OU DROIT DE SAVOIR?}

Quels sont les principes qui président en France au don de gamètes?

La gratuité du don (principe d'extra-patrimonialité du corps humain). Les donneurs ne sont donc pas rémunérés et doivent venir un certain nombre de fois, être reçus par les biologistes et les psychologues et faire plusieurs dons. La gratuité du don traduit un attachement à des valeurs éthiques extrêmement valorisées à la fois par les donneurs et les receveurs. Le couple donne à un autre couple, ayant déjà au moins un enfant. L'idée est qu'un couple doit pouvoir se représenter le fait d'être parent avant de donner ses gamètes. Une limitation du nombre d'enfants qu'un donneur peut engendrer par cette pratique vise à éviter que de trop nombreux enfants soient issus du même donneur afin d'éviter les rencontres éventuelles entre enfants partiellement consanguins.

L'autre principe est donc l'anonymat visant à préserver l'intimité de la vie du donneur et permettre aux parents reconnus comme tels de se sentir fondés à l'être. Les débats actuels ont le mérite de soulever la question de la place de l'enfant, jusqu'alors totalement occultée, tandis qu'un certain nombre de couples s'engageant dans la demande de dons de gamètes sont prêts à accepter la levée de l'anonymat et à en envisager les conséquences.

Pourtant, depuis les années 70, la tendance était plutôt de taire le don de spermatozoïdes ou de le révéler très tardivement. Les couples sachant que les secrets sont lourds à porter et parfois conscients de leur filtrage émotionnel, aspirent à une plus grande transparence envers leurs enfants sur le mode de procréation et déclarent de plus en plus leur intention de les en informer. Certains ont déjà révélé le don à leurs enfants lorsqu'ils viennent faire une deuxième ou troisième demande au CECOS pour des puînés. Une question récurrente est de savoir comment leur raconter leur «histoire» pour éviter la lourdeur de ce secret. Comment leur dire, quand leur dire? Mais aussi quoi leur dire quand ils ne savent pas si ces enfants pourront avoir connaissance d'informations sur le donneur. Face à cette épée de Damoclès sur la levée de l'anonymat, certains couples font aussi machine arrière et hésitent à informer l'enfant.

D'après Gérard Moutel du Laboratoire d'éthique médicale et de médecine légale de la Faculté de médecine Paris Descartes, (2009), la rupture de 1'anonymat fragilise le concept de gratuité : le concept de gratuité (issu de la notion de liberté et d'abolition de l'esclavage) ne doit pas être entendu forcément en termes financiers mais en termes de ce qui est donné sans contrepartie, sans échange. La levée de l'anonymat entraînerait un système de dépendance et de pressions potentielles à long terme entre donneur et receveur. Elle remettrait en cause le fait que ce dernier a pour but «d'éviter de délicats problèmes relationnels entre les personnes concernées ». Ainsi, d'après Gérard Moutel (2009), changer les règles reviendrait à changer les symboliques du don et la nature du contrat social et les conceptions que l'on a de la protection des personnes. 
Les pionniers du don de gamètes comme Georges David et d'autres cliniciens défendent le principe de l'anonymat comme garant de la liberté et du bénévolat vu du côté des adultes. Ils se fondent sur les travaux du sociologue Titmuss dans les années 70 qui démontra les avantages très nets du don bénévole dans les sociétés contemporaines, tant au plan économique que social. Ensuite deux positions différentes semblent s'opposer: celle de Mauss, centrée sur les sociétés primitives et celle de Jacques Derrida.

Jacques Derrida (1999) défend la thèse selon laquelle le don n'implique pas une logique de réciprocité. Ce qui est alors essentiel, c'est la dimension métaphysique du don au sens où le don permet au receveur d'exister, d'être heureux et indépendant, seul. C'est l'objet du don que le receveur reçoit, pas le donneur. Dès lors, le secret du don est une arme face à l'ego qui permet d'éviter l'égoïsme et l'égocentrisme de la démarche (ne se connaissant pas, donneur et receveur n'attendent rien de plus l'un de l'autre et l'on ne se met pas en position d'en attendre quelque chose). Le don, dans sa valeur absolue, est un effacement de soi. À ce titre on peut (ou l'on doit) comme donneur rester caché et le secret est une barrière morale à toute tentative de transaction secondaire (de part et d'autre). Dans son texte sur le don, Jacques Derrida donne l'exemple non des dons de gamètes mais des dons d'organes, dans un contexte qui ne concerne pas l'enfant.

Mais, plus concrètement, s'agissant des relations avec un donneur vivant ou les proches d'un défunt, cette hypothèse de réciprocité ne peut être écartée si l'on considère la dimension imaginaire. C'est ainsi que pour certains auteurs (M. Mauss), il n'est pas surprenant que des études sociologiques sur le don, n'y voient pas un geste unilatéral, mais le conçoivent dans la perspective d'un retour, possible mais non obligatoire (le contre-don), et potentiellement soit bénéfique, soit préjudiciable. Mauss (1925) posait donc deux questions fondamentales: quelle place reste-t-il aux échanges par dons dans les sociétés modernes du fait de l'introduction envahissante du marché dans les transferts de biens et de services? Et aussi, s'il y a persistance d'échanges par don, ont-ils pris une nouvelle forme ? Ces questions soulèvent les conséquences à la fois de commercialisation progressive des produits du corps humain et du marché autour de la procréation.

Pour ceux qui souhaiteraient lever l'anonymat du don, les arguments se placent du côté de l'enfant, généralement oublié dans cette délicate opération. Au plan collectif, un débat sur la transparence s'impose face à un «biopouvoir » tout puissant qui déciderait à la place des individus. Il revient donc à la société de se montrer plus mature, pourquoi cacher une pratique acceptée ? Pour la majorité des défenseurs de la levée de l'anonymat, il s'agit moins de faire du donneur un parent, mais du donneur un acteur qui a permis d'être présent au monde (de vivre). En effet, celui qui n'a pas le droit de savoir subit une certaine violence selon Irène Théry (2009). La recherche d'une « vérité biologique » est 
certainement au centre des préoccupations, mais ouvre plus souvent à la recherche de nouvelles parentalités.

Pour Pierre Jouannet (2010), la légitimité des demandes individuelles n'est pas en cause, concernant ces personnes qui « souffrent incontestablement de leur situation. Ils racontent souvent des histoires malheureuses de révélations faites tardivement [...]. Ils sont presque toujours confrontés à un malaise, quand ce n'est pas à de véritables carences dans l'histoire filiale qui les unit à leur père. $\mathrm{Ne}$ sont-ce pas ces carences, en effet, qui peuvent les pousser à chercher ailleurs que dans leur famille leur raison d'être au monde»?

Mais ces personnes sont peu nombreuses à demander la vérité biologique ou à rencontrer les donneurs. Ces demandes légitimes auront certes un effet déstabilisant et les modalités de l'anonymat devraient trouver une réponse de la société afin que les protagonistes se sentent sécurisés et légitimés par des réponses collectives et non plus individuelles. Les conséquences de la possible levée de l'anonymat sont loin d'être simples: quelles relations leurs enfants pourraient avoir avec un donneur qu'ils pourraient éventuellement rencontrer, une fois majeurs? Quelle est donc la place du donneur qui, lui, n'a aucun projet exprimé envers cet enfant? Quelle responsabilité engage le donneur en donnant ses gamètes? La place de ce tiers donneur a besoin d'être historicisée dans la démarche subjective du projet parental, vécu comme l'étranger, l'amant, l'intrus, le donneur magnanime idéalisé.

Cette difficulté récurrente à cerner une technique visant à pallier les infertilités tient peut-être au niveau où est posée la question de la filiation et de l'engendrement. Pourtant, alors que les sciences humaines n'ont jamais été aussi mêlées aux questions médicales, de rares biologistes, philosophes, anthropologues, juristes insistent sur la dimension symbolique dans la filiation et la procréation.

Les positions, peu nuancées, sans recul historique semblent s'opposer: soit à faire valoir le biologique dans la procréation alors que la filiation repose sur des fondements sociétaux, psychiques et juridiques; ou bien en voulant privilégier des liens familiaux purement fondés sur le «désir d'enfant» et sur le social et l'éducatif. Le sexuel et l'inconscient ne sont qu'indirectement invités au débat et l'on tourne en rond. Or, les conséquences de cette pratique courante sont toujours discutées et les enjeux juridiques, médicaux et psychiques toujours questionnés.

En effet, le désir d'enfant mobilise des représentations préconscientes et inconscientes, des fantasmes, des théories sexuelles infantiles à l'origine du désir de donner la vie. Comment anticiper cette naissance lorsqu'on est blessé par l'impossibilité à assurer une descendance et un lien de continuité avec ses ascendants?

Or, l'histoire du don de gamètes a toujours fait l'objet de discussions et la discrétion des médecins soucieux de respecter l'intimité des couples ayant commencé à pratiquer les dons de gamètes ne facilite pas la compréhension de ses enjeux. L'absence de réponses satisfaisantes impose de décaler quelque peu 
la perspective. Pourtant, cette technique assez simple ne pose pas de difficultés d'ordre médical. En 1776, Spallanzani réalise une fécondation artificielle sur des œufs de grenouille et démontre le caractère indispensable de la semence mâle. Il sera le premier à réaliser une fécondation artificielle chez l'animal. Son ami le philosophe et naturaliste C. Bonnet (1720-1793) lui écrit alors, d'une manière très prémonitoire: «Je ne sais même si ce que vous venez de découvrir n'aura pas quelque jour dans l'espèce humaine, des applications auxquelles nous ne songeons point et dont les suites ne seraient pas légères. »

L'application à l'homme ne tarde pas. En effet, en 1791, J. Hunter insémine la femme d'un drapier avec le sperme de ce dernier, et obtient une grossesse. L'IAD en tant que telle remonterait officiellement à 1884 (première tentative réussie William Pancoast aux États-Unis).

Au début du XIX ${ }^{\mathrm{e}}$ siècle, la technique est condamnée par l'Académie des Sciences morales et politiques (acte antinaturel qui posera des questions insolubles). Aujourd'hui, la logique du droit de la filiation semble aujourd'hui mise en question.

Pour les anthropologues, la filiation a un fondement social qui prime sur le biologique. La filiation est donc fondée sur l'ordre social en fonction de règles que la société s'est fixées. Elle est basée sur la différenciation entre les sexes, une rencontre sexuelle est nécessaire ainsi que la différence entre les générations, les individus sexués deviennent parents, mais il existe de nombreux systèmes de filiations différents: systèmes cognatique, matrilinéaire... L'advenue à l'existence de l'enfant est déterminée par le système de filiation de la société où il naît.

Pour les psychanalystes, l'enfant est investi par ses parents et crée de l'affiliation pour eux (Lebovici) lorsqu'il s'inscrit dans le maillage narcissique des générations qui l'ont précédé, le juridique institue le cadre de la filiation qui fait tenir ensemble à la fois le plan individuel et collectif.

Pierre Legendre (1993), à la fois juriste et psychanalyste, fait appel à l'institution juridique de la filiation qui se réfère à la Loi permettant de passer de l'ordre juridique à l'ordre symbolique. L'état est l'organisateur dans les sociétés occidentales de cet ordre qui permet la reproduction. Ainsi, le sujet individuel n'est pas auto référencé, mais fait partie d'un ordre symbolique à travers le langage. Pour Pierre Legendre, l'état garantit dans les sociétés occidentales un principe de droit civil des filiations. Lorsque le sujet se met en position d'auto fondation narcissique, il n'est pas à sa place. L'État est la référence pour différencier, pour théâtraliser la problématique de l'inceste, pour montrer la raison des lois... La Loi en formalisant le réel par une configuration symbolique permet de passer de l'individuel à des valeurs universelles : du vivant à l'homme, de l'être humain à l'être social inscrit dans une filiation. Pour Legendre, il y a un danger du discours de la science ou du scientisme lorsqu'elle se trouve en position d'autorité à la place du tiers mythique. 
Ainsi, peut-on définir avec Jean Guyotat (2005) le lien de filiation comme étant « ce par quoi un individu se relie et est relié, par le groupe auquel il appartient, à ses ascendants et descendants réels et imaginaires. C'est le groupe auquel le sujet appartient»; le groupe auquel il appartient le nomme «fils de » ou «père de» et le groupe plus élargi le désigne par le nom, le patronyme, dont on connaît bien sûr l'importance. Ce problème de patronyme est très discuté. C'est dire que le lien de filiation est extrêmement lié à l'organisation culturelle, à la signification que la culture donne à l'organisation de la société.

À partir des distinctions freudiennes de l'amour narcissique et de l'amour d'objet qu'il prolonge, Jean Guyotat distingue le lien de filiation instituée, permettant de se définir à partir d'un réseau symbolique, et la filiation narcissique visant à la reproduction du même. La filiation instituée est issue du droit, qui lui donne son ossature et définit la place de fils, la place de père, avec évidemment la transmission du nom mais aussi la transmission des biens, l'autorité parentale et l'appartenance. Ces repères symboliques confèrent des sens, des significations au fait qu'un père ou une mère, un enfant, au fil des jours, dans la réalité quotidienne de la relation, nomment, d'un même nom, un état émotionnel. C'est une façon de nommer, en commun, des états affectifs que l'enfant reprend ensuite pour son propre compte, pour les nommer lui-même. Cette relation entre le père et la mère, instituée par le langage, peut être décrite en termes affectifs mais aussi en termes de liens de filiation. On peut donc parler d'institutions langagières, mais il y a aussi les institutions non-langagières, la plus évidente étant, par exemple, la transmission des biens.

La filiation narcissique, quant à elle, offre une autre logique des liens. Elle est intéressante à considérer parce que c'est peut-être à travers elle que l'on peut comprendre la transmission des traumatismes. Ce système est un fantasme qui est une défense contre la mort : il s'agit d'un fantasme d'immortalité. Il y a une sorte de transmission, en direct, qui n'est plus modélisée par le symbolique de la filiation instituée. En résumé, l'enfant dispose pour se construire d'une filiation symbolique s'étayant sur un cadre juridique (filiation instituée) qui nomme le parent qui confère un sens à ses paroles et organise un maillage narcissique (filiation affective, imaginaire). Cette double assise parentale diachronique permet que l'enfant rencontre les effets de la névrose infantile transitoire et des identifications œdipiennes triangulées qui permettent d'intérioriser les différences des sexes et des générations et les interdits qui les accompagnent. L'enfant peut déployer ses mouvements tendres et agressifs et traverser les étapes de la sexualité infantile en surmontant ses conflits et complexifier le théâtre de ses relations intériorisées et avec autrui. Les réponses qu'il reçoit de l'adulte aux questions qu'il se pose avec le plus grand naturel sont les fruits des observations de son entourage et de leurs relations, marquées par les constructions qu'il échafaude à partir de ses 
fantasmes, de son imaginaire et de ses pulsions. Ces déductions, inférences, contradictions, l'aident à fortifier son narcissisme et à se forger sa propre conception du monde.

\section{DÉPASSER LE BIOLOGIQUE PAR LA PAROLE}

Nous rencontrons au CECOS des parents blessés imaginairement par cette impossibilité de transmettre la vie à des enfants qu'ils désirent. Procréer renvoie à des valeurs extrêmement diverses culturelles, identitaires, sociales, de transmission des biens matériels, de connaissances, de savoir-faire, de la nécessité de conserver l'espèce. Le souhait d'enfantement comprend également des valeurs sociales comme l'inscription dans la société à travers le regard des autres, la famille, le couple, l'accession à l'âge adulte face à ses propres parents, la recherche d'un compagnon pour vivre et d'un père et la reconnaissance à travers la maternité. Le désir d'enfant renvoie aussi à des valeurs personnelles de choix existentiels préconscients, comme repousser les craintes de la mort, s'incarner en l'autre, prolonger son narcissisme, retrouver son enfance et la rejouer autrement selon ses propres choix. Si devenir enceinte et mettre au monde un enfant sont des actes concrets, le désir et l'attente de l'enfant demeurent bien abstraits, fantasmés, marqués par la pensée magique et symbolisés de façon variée dans toutes les cultures. La naissance mobilise les attentes des couples, les croyances, les comportements face au cycle vital imaginaire, la conscience du temps, la crainte du nouveau-né, la peur de la mort. Le désir d'enfant relance les enjeux psychiques interpersonnels du couple et triangulés autour du bébé à venir, du garçon, de la fille, du féminin, du phallique, de la castration, du maternel, du paternel, et des autres ascendants. À ceci s'ajoute la notion de contrat narcissique proposée par Piera Aulagnier (1975), en référence à la double existence de l'individu soulignée par Freud, en tant qu'il poursuit sa propre fin et en tant qu'il est membre d'une chaîne. Ce sont toutes ces attentes qui semblent suspendues lors du constat de l'infertilité ce qui explique son caractère traumatique, comme si l'avenir semblait bouché et vide. Les couples sont parfois prêts à tout pour surmonter cette crise existentielle de la procréation, cette dernière apparaissant parfois comme une valeur refuge

À travers les entretiens cliniques, les couples infertiles expriment la souffrance de leur isolement et de leur solitude, repliés sur leurs problèmes qu'ils hésitent à partager avec leur entourage, surtout s'ils veulent maintenir le secret de leur démarche. Les conjoints sentent la menace de la pérennité de leur relation et ils ont souvent pensé s'exclure, se séparer. Ils connaissent les mouvements de tonalité dépressive, d'agressivité de celui qui est touché dans sa capacité à procréer ou une stigmatisation du conjoint infertile ou au contraire la surprotection du partenaire. La fluidité de la communication s'épaissit. Ces mouvements 
successifs d'espoir et de déception découragent et la solution médicale semble protéger les sujets d'un véritable effondrement. Toute élaboration du trauma est difficile et la proposition de don de gamètes permet de s'engouffrer dans l'espoir d'une pseudo guérison. Un temps d'attente du don est nécessaire à l'élaboration du deuil de la fertilité. L'hésitation à accepter immédiatement le don est plutôt le signe d'une élaboration de ce deuil. Or, le traitement médical trop rapidement accepté parait recouvrir ce qui est dévoilé par l'infertilité. En fait, la blessure de l'infertilité est une plaie jamais vraiment refermée, comme certains parviennent à l'avouer. Le désir d'enfant avec sa complexité et ses ambivalences se transforme parfois en véritable obsession d'un enfant à tout prix malgré les échecs qui ne sont pas rares quand l'infertilité est partagée. À travers le désir d'enfant non réalisé s'expriment des problématiques œdipiennes réactivées («avoir l'enfant du père » «ou enfanter de la mère ») ou bien des problématiques préœdipiennes régressives visant à retrouver l'objet premier d'amour. D'autres deuils plus anciens, d'autres histoires familiales souffrantes sont réactivés. Les couples ne peuvent élaborer la dette de vie qu'ils imaginent contractée avec les figures des images parentales dont ils restent toujours tributaires, éternels enfants qui ne peuvent se considérer vraiment comme adultes. L'intériorisation de ces conflits est si douloureuse qu'il faut du temps pour y parvenir. L'absence de symbolisation des représentations autour du deuil de la transmission filiative à travers la reproduction naturelle confine à l'émoussement des affects, des relations sexuelles, à la pensée opératoire et au refoulement des conflits. Ils cherchent une réassurance à travers des réponses trouvées sur internet ou bien savoir si les cliniciens vont pouvoir y répondre comme ils l'ont imaginé, ils se déclarent prêts à accepter un enfant réparateur de leur narcissisme blessé.

Dans la mesure où le parcours pour devenir parent est long, pénible, semé d'embûches, le découragement est possible, mais aussi une persévérance excessive, voire un certain acharnement. Des couples sont prêts à tout tenter pour avoir un enfant. Goëb et Al. (2004) ont souligné la lourdeur des différents temps des prises en charge médicales de l'infertilité (attente de la grossesse naturelle, temps de l'investigation, de la procédure, des répétitions, des suites...) entrâ̂nant espoir, anxiété, déception si échec, tension de couple, médicalisation, anxiété si succès... Ainsi, en dehors des fragilités individuelles, le parcours du traitement de la stérilité pourrait, en soi, être déstabilisant.

Les troubles liés à la difficulté de procréer constituent une souffrance reconnue de tous. Des travaux restent à mener sur les conséquences psychologiques de ces techniques (le deuil de la fertilité, la façon dont les enfants perçoivent la PMA, la révélation ou non de leur mode de conception). (Lazaratou et Golse, 2006).

Peut-être manque-t-on encore de recul, de critères collectifs et d'exemples pour se rendre compte de l'impact psychologique des procréations médicalement assistées ; par souci de respect de la discrétion et de préservation de l'ano- 
nymat, on ne connaît pas précisément, le devenir des enfants nés par insémination avec donneur. Les psychanalystes n'ont pas par vocation à répondre à la question de la suppression ou non de l'anonymat, sauf d'un point de vue citoyen, mais ils peuvent contribuer au débat en favorisant le travail psychique autour de l'infertilité comme une épreuve que les couples devront surmonter.

\author{
Claire SQUIRES \\ 45, rue du Faubourg-Montmartre \\ 75009 Paris \\ clairesquires@orange.fr
}

\title{
Bibliographie
}

Ameisen J.-C., Journées annuelles d'éthique 2011 du Comité Consultatif National d'Éthique, 22 janv. 2011.

sites.radiofrance.fr/franceinter/em/sur-les.../index.php

Aulagnier, P., La Violence de l'interprétation (1975), Paris, PUF, 1986.

Derrida, J., L'éthique du don, L'Animal autobiographique, colloque de Cerisy, Galilée, Paris, 1999

Epelboin, S., «Assistance médicale à la procréation. Principes et réflexions cliniques », in Espaces éthiques, pratiques hospitalières et lois de bioéthique. Perspectives de révision des lois du 29 juillet 1994, APHP, 1999, p. 93-98.

Gauchet, M ., «Mutations dans la famille», La revue lacanienne., 2010/3 - nº 8 pages 17 à 32, Paris, Erès.

Goëb, J.L., Férel S., Guetta J., et al.: «Vécus psychologiques des démarches d'assistance médicale à la procréation », Annales Médico-Psychologiques, 2006 ; 164: 781-788.

Guyotat, J., Transmission. Filiation, L'Esprit du temps, Recherches en psychanalyse, 2005/1, $\mathrm{n}^{\circ} 3, \mathrm{p} .115-119$.

Jouannet, P., Mieusset R., Donner et après... la procréation par don de spermatozoïdes avec ou sans anonymat? Springer, Paris, 2010.

Lazaratou, H., Golse B.: «Du désir à l'acte: les enfants de la procréation médicalement assistée (PMA)», Psychiatrie de l'Enfant, 2006; 49: 573-599.

Lebovici, S., Diatkine, R., Soulé M., Nouveau traité de psychiatrie de l'enfant et de l'adolescent (4 volumes), éd. PUF, 2004.

Legendre, P., Les enfants du texte, Étude sur la fonction parentale des États, Paris, Fayard, 1993.

Lévy-Soussan, P., «Travail de filiation et adoption », Revue française de psychanalyse 1/2002 (Vol. 66), p. 41-69.

Mauss, M., Essai sur le don. Forme et raison de l'échange dans les sociétés archä̈ques (1925), Introduction de Florence Weber, Quadrige/Presses universitaires de France, 2007.

Memmi, D., « Sonder les âmes ou radiographier les corps ?», in IACUB M., JOUANNET P. 
(éds): Juger la vie, La découverte, Paris, 2001.

Moutel, G., Médecins-patients, l'exercice de la démocratie sanitaire, Éd. L'Harmattan, 2009. Squires, C., et al., «Psychopathologie et procréation médicalement assistée: Comment les couples infertiles élaborent-ils la demande d'enfant?», Devenir 2/2008 (Vol. 20), p. 135149.

Thery, I., L'anonymat des dons d'engendrement est-il vraiment «éthique »? Revue Esprit, mai 2009.

\section{Claire Squires - Essai sur le don de gamètes}

Résumé : La possible levée de l'anonymat des donneurs dans le cadre des procréations médicalement assistées avec insémination des spermatozoïdes ou fécondation d'un ovocyte d'un tiers est débattue en France. Elle s'inscrit dans le courant initié par des recherches en paternité ou en maternité visant à la «traçabilité » des origines et du «droit » des enfants à connaître leurs origines. Ces filiations électives relancent le débat nature/culture et les représentations qui les accompagnent dans le domaine de la médecine de la reproduction. La technique semble parfois dépasser les possibilités psychiques de supporter les conséquences de l'infertilité.

Mots-clés : Don de gamètes - Anonymat - Filiation - Lois de bioéthique - Origines.

\section{Claire Squires - An Essay on Gamete Donation.}

Summary : The question of lifting the anonymity of donors in medically assisted procreation procedures, involving artificial insemination with sperm donation or fertilisation of an oocyte donated by a third party, is at present the subject of heated debate in France. It is rooted in a train of thought underpinned by children trying to trace their paternal or maternal filiation and aims at making this easier, thereby promoting the child's 'right' to gain knowledge of their own origins. Elective filiations such as these refuel the nature/nurture debate and the representations they go hand in hand with within the framework of reproductive medicine. Technical possibilities sometimes seem to far outdo the psychic capacity to cope with the consequences of infertility.

Key-words : Gamete donation - Anonymity - Filiation - Bioethics Law - Origins. 